\title{
Suppressing Speckle Noise for Simultaneous Differential Extrasolar Planet Imaging (SDI) at the VLT and MMT
}

\author{
Beth A. Biller ${ }^{1}$, Laird M. Close ${ }^{1}$, Rainer Lenzen ${ }^{2}$, \\ Wolfgang Brandner ${ }^{2}$, Donald McCarthy ${ }^{1}$, Eric Nielsen ${ }^{1}$, \\ Stephan Kellner ${ }^{2}$, and Markus Hartung ${ }^{3}$ \\ ${ }^{1}$ Steward Observatory, University of Arizona, Tucson, AZ 85721 \\ email: bbiller@as.arizona.edu \\ ${ }^{2}$ Max-Planck-Institut für Astronomie, Königstuhl 17, 69117 Heidelberg, Germany \\ ${ }^{3}$ European Southern Observatory, Alonso de Cordova 3107, Santiago 19, Chile
}

\begin{abstract}
We discuss the instrumental and data reduction techniques used to suppress speckle noise with the Simultaneous Differential Imager (SDI) implemented at the VLT and the MMT. SDI uses a quad filter to take images simultaneously at 3 wavelengths surrounding the $1.62 \mu \mathrm{m}$ methane bandhead found in the spectrum of cool brown dwarfs and gas giants. By performing a difference of images in these filters, speckle noise from the primary can be significantly attenuated, resulting in photon noise limited data. Non-trivial data reduction tools are necessary to pipeline the simultaneous differential imaging. Here we discuss a custom algorithm implemented in IDL to perform this reduction. The script performs basic data reduction tasks but also precisely aligns images taken in each of the filters using a custom shift and subtract routine. In our survey of nearby young stars at the VLT and MMT (see Biller et al., this conference), we achieved $\mathrm{H}$ band contrasts $>25000(5 \sigma \Delta \mathrm{F} 1(1.575 \mu \mathrm{m})>10.0 \mathrm{mag}, \Delta \mathrm{H}>11.5 \mathrm{mag}$ for a T6 spectral type object) at a separation of 0.5 " from the primary star. We believe that our SDI images are among the highest contrast astronomical images ever made from ground or space for methane rich companions.
\end{abstract}

Keywords. instrumentation: adaptive optics, methods: data analysis, techniques: image processing, (stars:) planetary systems.

\section{Introduction}

Adaptive optics (AO) systems that are "photon noise limited" can detect an object up to $10^{5}$ times fainter its primary at separations $>1$ " - sufficient to detect a young, selfluminous giant extrasolar planet. However, the contrast problem isn't the only obstacle in directly detecting extrasolar planets - Beyond the contrast limit problem, all AO systems suffer from a limiting "speckle noise" floor (Racine et al. 1999). Within 1" of the primary star, the field is filled with speckles left over from instrumental features and residual atmospheric turbulence after adaptive optics correction. These speckles vary as a function of time and color. For photon noise limited data, the signal to noise $\mathrm{S} / \mathrm{N}$ is proportional to $t^{0.5}$, where $t$ is the exposure time. For speckle-noise limited data, the $\mathrm{S} / \mathrm{N}$ does not increase with time past a specific speckle-noise floor (limiting contrasts to $\sim 10^{3}$ at 0.5 "). This speckle-noise floor is considerably above the photon noise limit and makes planet detection very difficult. Interestingly, space telescopes such as HST also suffer from a similar limiting speckle-noise floor due to imperfect optics and "breathing" (Schneider et al. 2003). Direct detection of extrasolar giant planets requires special instrumentation to suppress this speckle noise floor and produce photon noise limited images. 

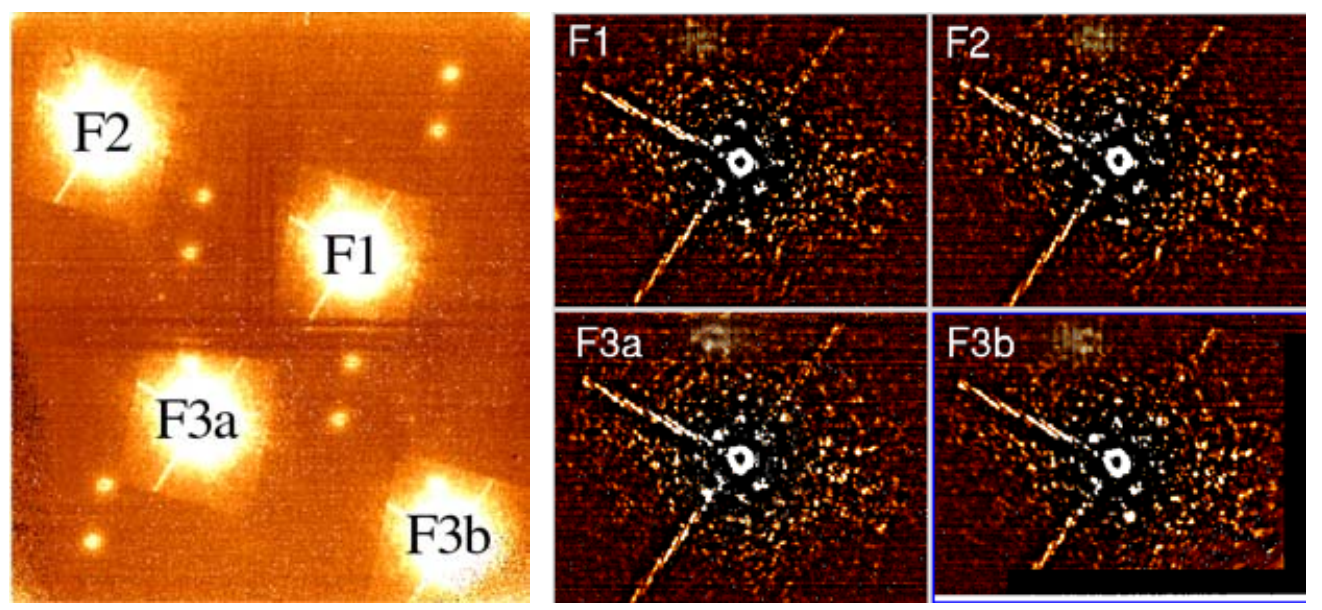

Figure 1. Left: Two minutes of raw SDI data from NACO SDI's $1024 \times 1024$ Aladdin array in the CONICA AO camera (Lenzen et al. 2004). Right: Same dataset, slightly processed. Apertures have been selected around each filter image. In order to reveal the speckle pattern, a heavily smoothed image was subtracted from the raw images (unsharp masking). The resulting speckle patterns are very similar between the 4 simultaneous images which means that an effective subtraction of speckles can be obtained between the filters.

Simultaneous Differential Imaging is an instrumental method which can be used to calibrate and remove the "speckle noise" in AO images, while also isolating the planetary light from the starlight. This method was pioneered by Racine et al. (1999), Marois et al. (2000), Marois et al. (2002) and Marois et al. (2005). It exploits the fact that all cool $\left(\mathrm{T}_{\text {eff }}\right.$ $<1200 \mathrm{~K}$ ) extra-solar giant planets are thought to have strong $\mathrm{CH}_{4}$ (methane) absorption redwards of $1.62 \mu \mathrm{m}$ in the $\mathrm{H}$ band infrared atmospheric window (Burrows et al. 2001, Burrows et al. 2003). Our SDI device obtains four images of a star simultaneously through three slightly different narrowband filters (sampling both inside and outside of the $\mathrm{CH}_{4}$ features). These images are then differenced. This subtracts out the halo and speckles from the bright star to reveal any massive extrasolar planets orbiting that star. Since a massive planetary companion will be brightest in the F1 $1.575 \mu \mathrm{m}$ filter and absorbed in the rest, while the star is bright in all three, a difference can be chosen which subtracts out the star's light and reveals the light from the companion. Thus, SDI also helps eliminate the large contrast difference between the star and substellar companions.

\section{The SDI AO Cameras at the VLT and MMT}

An SDI imaging scheme is currently implemented at the 6.5m MMT (using the MMT AO adaptive secondary mirror and the ARIES AO camera - McCarthy et al. 1998) and at the ESO VLT (using the 8m UT4 and the NAOS-CONICA (NACO) AO system) by a group headed by L. Close and R. Lenzen (Close et al. 2005, Lenzen et al. 2004, Lenzen et al. 2005). Both devices are fully commissioned and available for observing.

The SDI technique requires some specialized optics consisting of a cryogenic custom double calcite Wollaston device and a focal plane quad $\mathrm{CH}_{4}$ filter. Our custom Wollaston splits the beam into four identical beams while minimizing non-common path errors. The differential non-common path errors are less than $10 \mathrm{~nm}$ RMS per Zernike mode (Lenzen et al. 2004). Each of the four beams is fed through one of the filters on the quad filter. Filter wavelengths were chosen on and off the methane absorption feature at $1.62 \mu \mathrm{m}$ and were spaced closely (every $0.025 \mu \mathrm{m}$ ) in order to limit residuals due to speckle and 

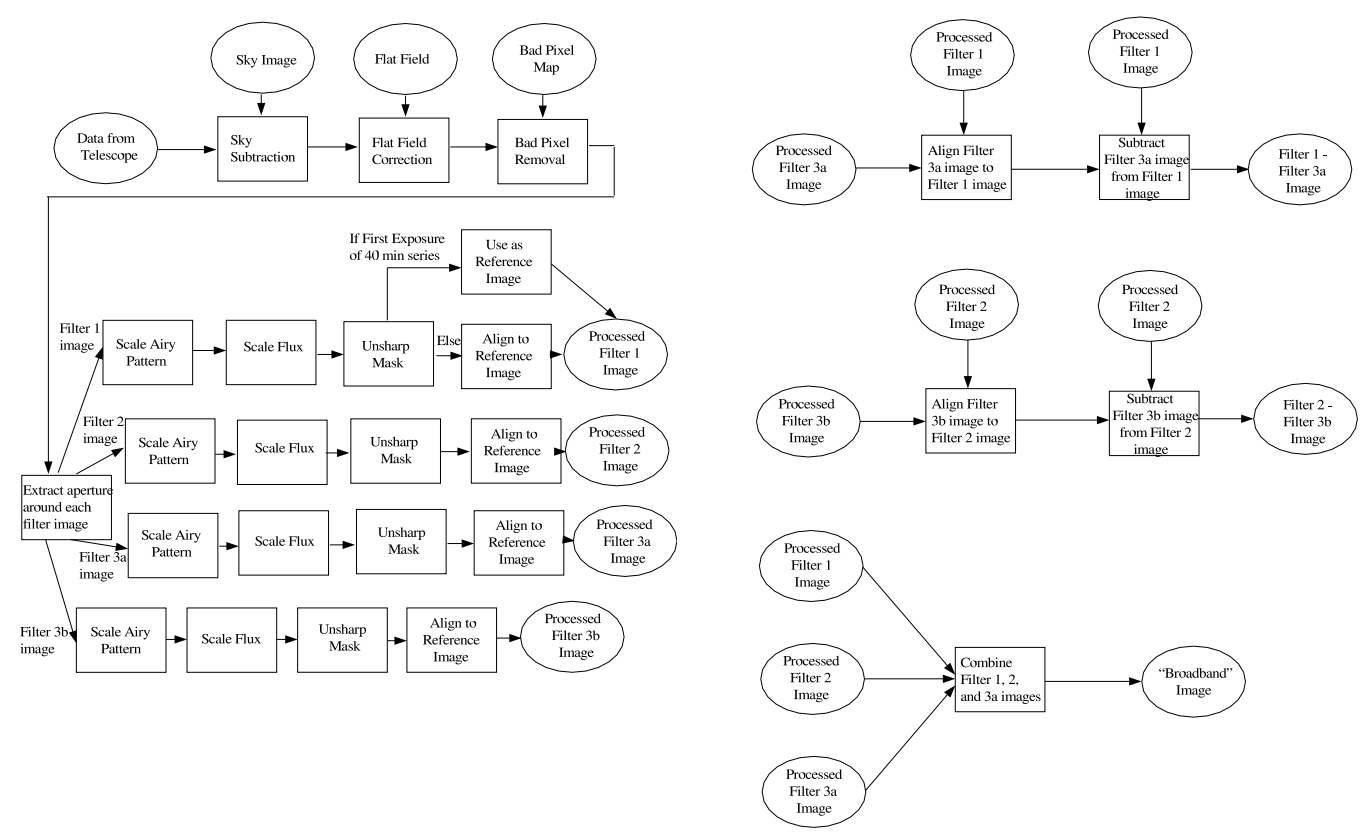

Figure 2. Pipeline Block Diagrams.

calcite chromatism. We used four filters F1, F2, F3a, and F3b with central wavelengths $\mathrm{F} 1=1.575 \mu \mathrm{m}, \mathrm{F} 2=1.600 \mu \mathrm{m}$, and $\mathrm{F} 3 \mathrm{a}=\mathrm{F} 3 \mathrm{~b}=1.625 \mu \mathrm{m}$. The filters are approximately $0.025 \mu \mathrm{m}$ in bandwidth. A cold $5 " \times 5 "$ focal plane mask has been implemented as a field stop for the VLT device. No coronagraph is currently used, since the Strehl ratios $(\sim 20$ $30 \%)$ are too low to increase the contrasts significantly. The SDI camera has a platescale of $0.017^{\prime \prime} /$ pix.

\section{Observational Technique and Data Reductions}

A raw dataset from NACO SDI is shown in Fig. 1. The inner 0.2" diameter core is saturated in each image to increase signal in the halo. After unsharp masking, we find that the speckle patterns in each of the separate filters are nearly identical. (See Fig. 1). This bodes well for our ability to attenuate speckle noise.

To distinguish between faint planets and any residual speckles, we observe each object at a variety of position angles. Instrumental and telescope "super speckles" (Racine et al. 1999) should not rotate with a change of rotator angle; however, a real planet should appear to rotate by the change in rotator angle. The data is reduced using a custom IDL script. A pipeline block diagram for this IDL script is presented in Fig. 2. Alignments are performed using a custom shift and subtract algorithm. We calculate 2 differences (and one non-differenced combination) which are sensitive to substellar companions of spectral types "T" ( $\left.\mathrm{T}_{\text {eff }}<1200 \mathrm{~K}\right)$, "Y" ( $\left.\mathrm{T}_{\text {eff }} \leqslant 600 \mathrm{~K}\right)$, and "L" ( $\left.\mathrm{T}_{\text {eff }}>1200 \mathrm{~K}\right)$. Data taken at different position angles are subtracted (e.g. 20 minutes of data at 0 degrees minus 20 minutes of data at 33 degrees) in order to further attenuate speckle noise.

A fully reduced dataset from the VLT SDI device as well as the same dataset reduced in a standard $\mathrm{AO}$ manner is presented in Fig. 3. Simulated planets were inserted into 

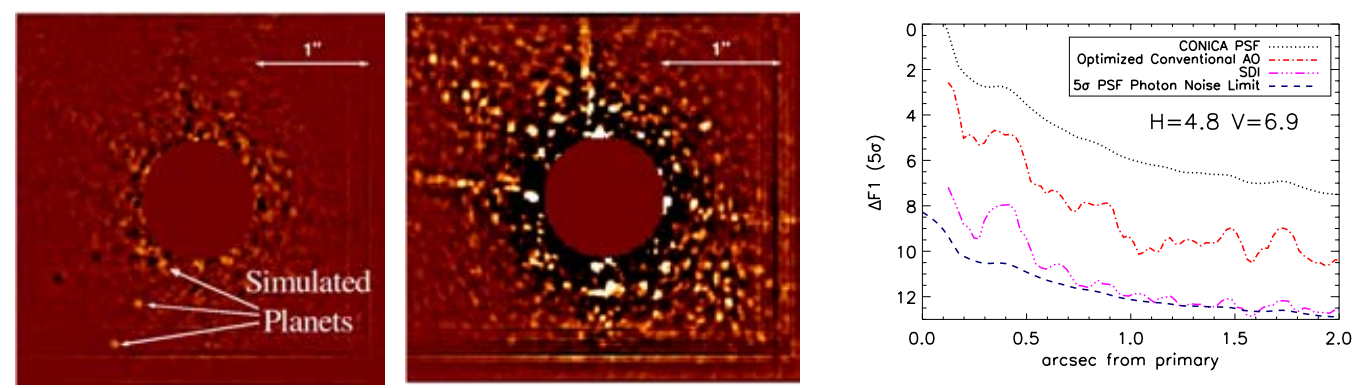

Figure 3. Left: A complete reduced dataset (40 minutes of data at a series of rotator angles $0^{\circ}, 33^{\circ}, 33^{\circ}, 0^{\circ}$ ) from the VLT SDI device. Simulated planets have been added at separations of $0.55,0.85$, and 1.35 " from the primary, with $\Delta \mathrm{F} 1=10 \mathrm{mag}$ (attenuation in magnitudes in the F1 $1.575 \mu \mathrm{m}$ filter) fainter than the primary. These planets are scaled from unsaturated images of the example star taken right before the example dataset (and have fluxes in each filter appropriate for a T6 object). Past 0.7", the simulated planets are detected with S/N $>$ 10. Center: Standard AO data reduction of the same dataset. Images have been coadded, flat-fielded, sky-subtracted, and unsharp-masked. Simulated planets have been added with the same properties and at the same separations as before. None of the simulated planets are clearly detected in the standard $\mathrm{AO}$ reduction. Additionally, numerous bright super speckles remain in the field. Right: $\Delta \mathrm{F} 1$ ( $5 \sigma$ attenuation in magnitudes in the $1.575 \mu \mathrm{m}$ F1 filter) vs. Separation for 40 minutes of VLT SDI data for a $70 \mathrm{Myr}$ K1V star at $15 \mathrm{pc}$. The top curve is the AO PSF. The next curve is the "classical AO PSF" unsharp masked. The third curve down is 40 minutes of SDI $0^{\circ}-33^{\circ}$ data. The last curve is the theoretical contrast limit due to photon-noise. At star-companion separations $>0.5$ ", we are photon-noise limited and achieve star to planet $\mathrm{H}$ band contrasts $>25000(5 \sigma \Delta \mathrm{F} 1(1.575 \mu \mathrm{m})>10.0 \mathrm{mag}, \Delta \mathrm{H}>11.5 \mathrm{mag}$ for a T6 spectral type object) at a separation of 0.5 " from the primary star.

Table 1. Properties of Example SDI Survey Stars and Comparison Stars.

\begin{tabular}{lcccccccc}
\hline Case & Spectral Type & Age & Distance & $\mathrm{H}$ & $\mathrm{V}$ & $\Delta \mathrm{F}^{1}$ & $\Delta \mathrm{H}^{1}$ & Exposure Time \\
\hline $\mathrm{A}$ & $\mathrm{K} 2 \mathrm{~V}$ & $30 \mathrm{Myr}$ & $45.5 \mathrm{pc}$ & 7.1 & 9.1 & 10.5 & 12 & $40 \mathrm{~min}$ \\
$\mathrm{~B}$ & $\mathrm{~K} 1 \mathrm{~V}$ & $70 \mathrm{Myr}$ & $15 \mathrm{pc}$ & 4.8 & 6.9 & 10.5 & 12 & $40 \mathrm{~min}$ \\
$\mathrm{C}$ & $\mathrm{M} 3 \mathrm{~V}$ & $30 \mathrm{Myr}$ & $24 \mathrm{pc}$ & 7.1 & 12.2 & 10 & 11.5 & $40 \mathrm{~min}$ \\
$10 \mathrm{~K}-\mathrm{M}$ stars $^{2}$ & $\mathrm{~K}-\mathrm{M}$ & $0-1 \mathrm{Gyr}$ & $10-50 \mathrm{pc}$ & $6.4-8.7$ & $8-12$ & 8.61 & 10.31 & $10-25 \mathrm{~min}$ \\
${\mathrm{Gl} 86^{3}}$ & $\mathrm{~K} 1 \mathrm{~V}$ & $10 \mathrm{Gyr}$ & $10.9 \mathrm{pc}$ & 4.2 & 6.2 & 12.8 & 14.3 & $80 \mathrm{~min}$ \\
\hline
\end{tabular}

${ }^{1} 5 \sigma$ at $0.5 "{ }^{2}$ Masciadri et al. IAUC $200{ }^{3}$ Mugrauer \& Neuhäuser 2005.

the dataset pre-reduction. In the SDI reduction, simulated planets (with $\Delta \mathrm{F} 1=10-5 \sigma$ attenuation in magnitudes in the $1.575 \mu \mathrm{m} \mathrm{F1} \mathrm{filter)} \mathrm{are} \mathrm{detected} \mathrm{with} \mathrm{S} / \mathrm{N}>10$ past 0.7". In comparison, none of the simulated planets are clearly detected in the standard AO data reduction and numerous bright super speckles remain in the field. A plot of $\Delta \mathrm{F} 1(5 \sigma)$ vs. separation from the primary is presented in Fig. 3. For this dataset, we achieved star to planet $\mathrm{H}$ band contrasts $(5 \sigma)>25000(5 \sigma \Delta \mathrm{F} 1(1.575 \mu \mathrm{m})>10.0 \mathrm{mag}$, $\Delta \mathrm{H}>11.5 \mathrm{mag}$ for a $\mathrm{T} 6$ spectral type object) at a separation of 0.5 " from the primary star - approaching the photon-noise limit in 40 minutes of data. $\Delta \mathrm{F} 1(1.575 \mu \mathrm{m})$ and $\Delta \mathrm{H}$ (for a methane object) for 3 of our survey stars (see Biller et al. this conference) as well as for two other comparison objects are shown in Table 1 - it is clear that the achievable contrast varies according to the magnitude of the object and total exposure time. We believe these are among the highest contrast astronomical images taken to date of methane rich companions. 


\section{Acknowledgements}

BAB acknowledges support through the NASA GSRP program. LMC acknowledges support through NSF CAREER and NASA Origins grants.

\section{References}

Burrows, A., Hubbard, W.B., Lunine, J., \& Liebert, J. 2001, Reviews of Modern Physics 73, 719

Burrows, A., Sudarsky, D., \& Lunine, J. 2003, ApJ 596, 587

Close, L.M., Lenzen, R., Biller, B.A., Brandner, W., \& Hartung, M. 2005, Science with AO ESO Workshop September 16-19 2003, 136

Lenzen, R., Close, L., Brandner, W., Hartung, M., \& Biller, B. 2004, Proc. SPIE 5492

Lenzen, R., Close, L., Brandner, W., Biller, B., \& Hartung, M. 2005, Science with AO ESO Workshop September 16-19 2003, 46

Masciadri, E. et al. 2006, IAUC 200 proceedings

Marois, C., Doyon, R., Racine, R., \& Nadeau, D. 2000, Proceedings of the Astronomical Society of the Pacific 112, 91

Marois, C., Doyon, R., Racine, R., \& Nadeau, D. 2002, Proc. SPIE 4008, 788

Marois, C., Doyon, R., Nadeau, D., Racine, R., Riopel, M., Vallée, P., \& Lafrenière, D 2005, Proceedings of the Astronomical Society of the Pacific, 117, 745

McCarthy, D., Burge, J., Angel, R., Ge, J., Sarlot, R., Fitz-Patrick, B., \& Hinz, J. 1998, Proc. SPIE 3354, 750

Mugrauer, M. \& Neuhäuser, R. 2005, MNRAS, 361, L15

Racine, R., Walker, G.A.H., Nadeau, D., Doyon, R., \& Marois, C. 1999, Proceedings of the Astronomical Society of the Pacific 111, 587

Schneider, G., Becklin, E., Close, L., Figer, D., Lloyd, J., Macintosh, B., Hines, D., Max, C., Potter, D., Rieke, M., Scoville, N., Thompson, R., Weinberg, A., \& Windhorst, R. 2003, solicited by STSCI in preparation for HST Cycle 12 

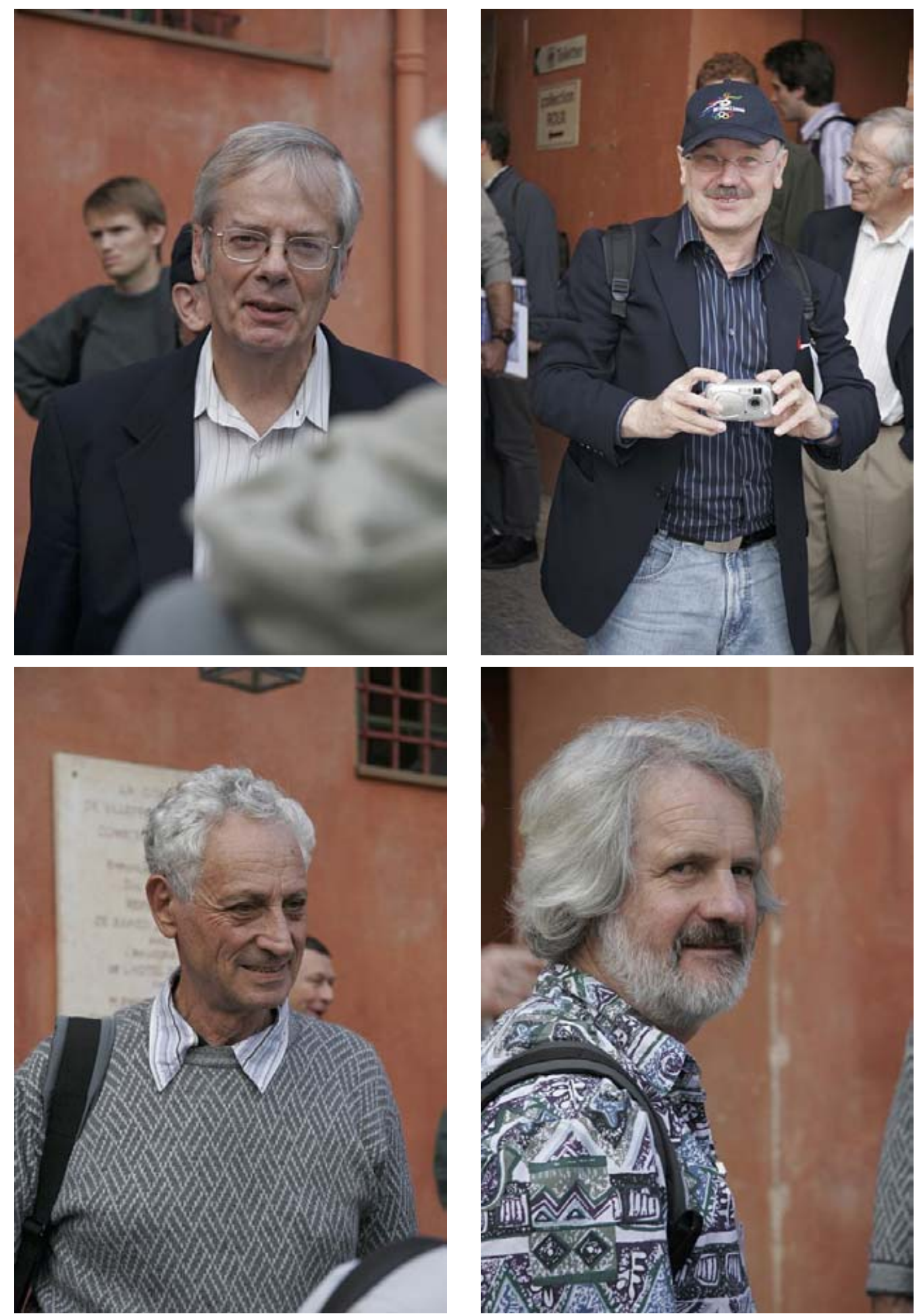

All photographs: Laurent Thareau [1.thareau@free.fr]. 